\title{
Proton and $x$-ray irradiation of silicon devices at the TIFPA-INFN facilities in Trento
}

\author{
Benedetto Di Ruzza ${ }^{a, *}$ \\ ${ }^{a}$ Trento Institute for Fundamental Physics and Applications (TIFPA-INFN), \\ Via Sommarive 14, 38123 Trento, Italy \\ E-mail: benedetto.diruzza@tifpa.infn.it
}

Proton and x-ray irradiation are essential procedures required to characterize the effects of TID and displacement damage designing silicon sensors for charged particle. The experimental area of the Trento Proton Therapy Center, a medical facility located in Trento (Italy), allows to perform proton irradiation on SiPMs, solid state silicon based (pixel, drift and microstrip) sensors and experimental electronic devices using protons with energy in the range of 70-230 $\mathrm{MeV}$. The irradiation isocenter is in air, the circular beam spot has a gaussian intensity profile with a sigma varying between $2.74 \mathrm{~mm}$ and $6.92 \mathrm{~mm}$ and a maximum proton rate of about $10^{\wedge} 10$ protons/s. For the energy of $120 \mathrm{MeV}$ a devoted set-up, called dual ring, allows to achieve a uniform beam spot on a circumference of $30 \mathrm{~mm}$ radius. This energy range is especially suitable for testing devices oriented to medical and space applications, but it is also useful for high-energy detector upgrades studies. In the TIFPA-INFN laboratories is also located a tungsten anode x-ray source allowing a TID characterization of experimental silicon devices. In this document will be given a description of the experimental area of the Trento Proton Therapy Center and some results of the proton and x-ray irradiation on prototype sensors for charged particle.

40th International Conference on High Energy physics - ICHEP2020

July 28 - August 6, 2020

Prague, Czech Republic (virtual meeting)

*Speaker 


\section{Introduction}

This article describes the latest proton and x-ray irradiation activities performed in the laboratories of the Trento Institute for Fundamental Physics and Applications (TIFPA) [1], the Trento Center of the Italian National Institute for Nuclear Physics (INFN)[2]

Proton irradiation are performed in the experimental area of the Trento Proton Therapy Center (TPTC)[3], that is a medical facility for hadron therapy located in Trento (Italy) and operated by the Azienda Provinciale Servizi Sanitari (APSS)[4].

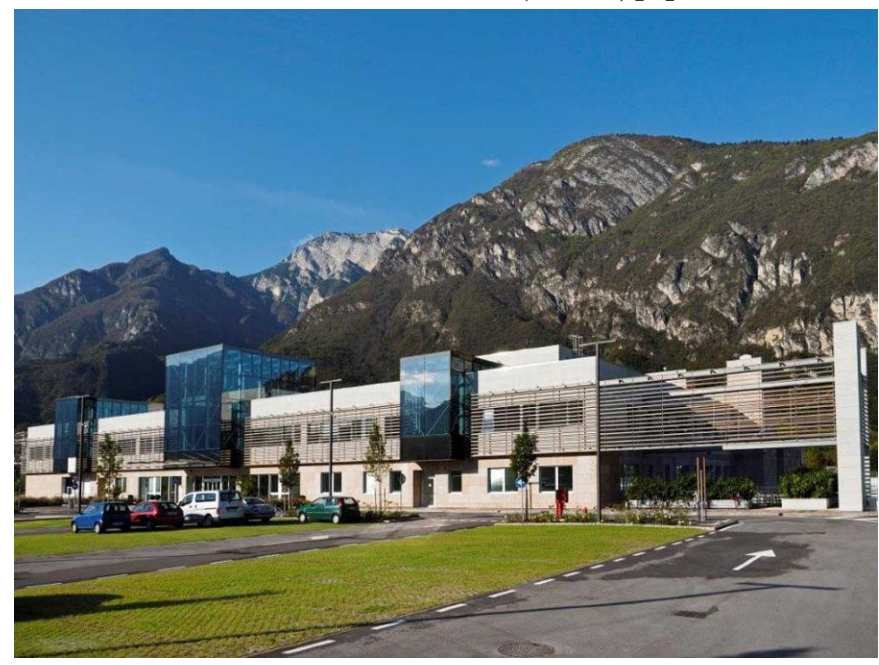

Fig. 1.1: The Trento Proton Therapy Center.

TPTC clinical activity started in 2014 and since then more than 1300 oncological patients were treated. The facility is equipped with two gantry rooms for patient treatment and a experimental area for physics and biophysics experiments. The experimental area is operated by the TIFPA-INFN [5] in collaboration with the APSS and IBA [6]. This area is used exclusively for non clinical activities studies such as physics, biophysics and radiological studies, tests and irradiations.

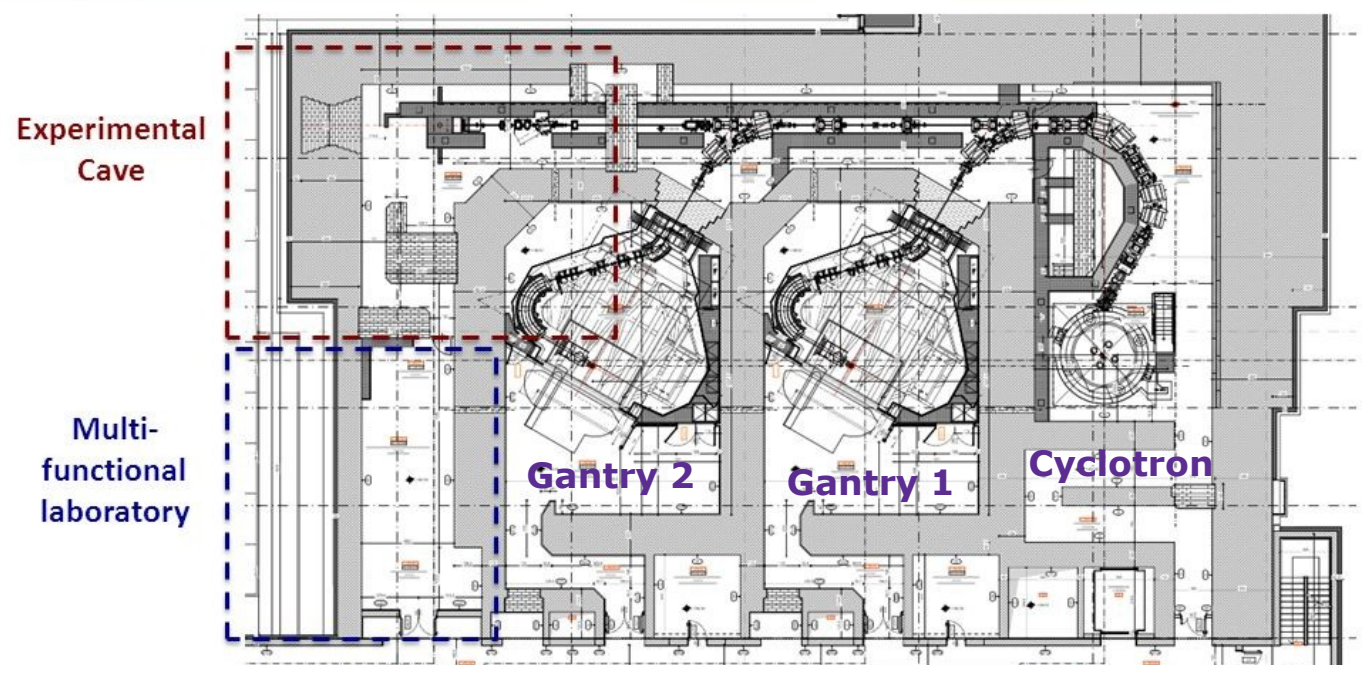

Fig. 1.2 : Trento Proton Therapy Center layout. 
The experimental area is divided in two parts: the experimental cave and the multifunctional laboratory, that is used also as cave remote control room during the irradiations and test beam activities.

TIFPA-INFN x-ray irradiations are performed in different laboratory, located in the TIFPA facility of the Povo site, close to the main building of the University of Trento Physics Department [7].

\section{Proton Irradiations}

Lattice displacement damage in solid state sensors due to Not Ionizing Energy Loss (NIEL) of incident particles, is responsible of performance degradation for dosimetric, traking and calorimetric systems. Proton irradiations is a simple and cheap way to perform characterization of sensors in order to predict these behavior. A medical proton facility, like the TPTC allows to perform these measurement for devices used in medical and space application.

\subsubsection{The TPTC Cyclotron}

The TPTC proton accelerator is a $106 \mathrm{MHz}$ cyclotron built and operated by the IBA Corporation. Protons are extracted at the energy of $230 \mathrm{MeV}$, then energy can be lowered up to $70 \mathrm{MeV}[3]$ using a degrader. Maximum extraction current is $320 \mathrm{nA}$. Proton rate can be tuned from $200 \mathrm{p} / \mathrm{s}$ up to $10^{\wedge} 10 \mathrm{p} / \mathrm{s}$.

\subsubsection{The Experimental area}

In the experimental cave of the experimental area two almost identical beamlines (called zero degree and thirty degree beamline) are available for in air experiments, test and irradiations. For practical reasons the zero degree is used mainly for biological experiments, while the thirty degree line is used mainly for physics activities.
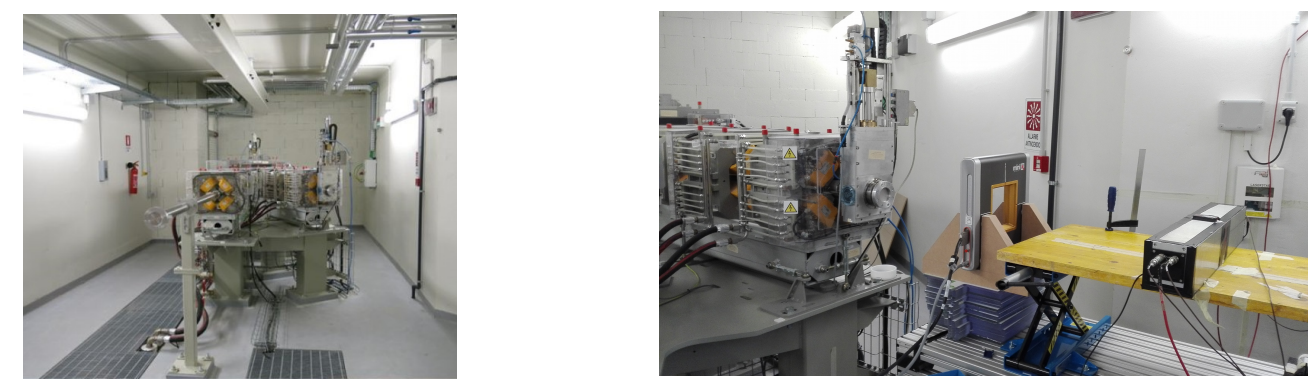

Fig 2.1: The two beamlines during the commissioning (left) and the thirty degree beamline during a test-beam (right).

The cave is equipped with a laser system that allows to identify beam position and the isocenters. A pach-panel with SHV, ethernet and BNC connections is also present and is connected with a corresponding panel located in the contiguous multi-functional laboratory. The beam in the two isocenters has a gaussin intensity profile with a energy dependent sigma as described in ref.[9]. Since the priority of the TPTC facility is medical treatment of oncological patient, the proton beam is available in the experimental area only after the end of daily clinical 
activities (that happen generally around 7.30 PM) up to $10.30 \mathrm{PM}$ in the working days and on Sunday morning from $8 \mathrm{AM}$ up to $2 \mathrm{PM}$.

For safety reasons there is the administrative limit of maximum $0.5 \mathrm{mC}$ proton beam charge as maximum achievable amount of delivered protons during a day of activity, working at the maximum nominal current of $320 \mathrm{nA}$ (nominal current measured at the cyclotron exit) this limit is achieved in 20 minutes. Furthermore, working at nominal currents greater than $100 \mathrm{nA}$, due to technical interlocks restrictions, irradiation have to be paused for 5 minutes after every 5 minutes of irradiation. After the work all the irradiated material is checked using a cooled germanium detector and only if the the measured activity is below the administrative limits, can be brig back by the users.

\subsubsection{Direct Beam Irradiations}

For small sensors and devices, such as SiPM or test structures, irradiation can be performed putting directly the sensor in the beam isocenter. In this conditions is possible to perform the irradiation at the maximum beam intensity, but the gaussian profile of the beam have to be considered in the evaluation of the delivered beam.

\subsubsection{Dual Ring Irradiations}

Since a beam pencil with a gaussian intensity profile is useful for tracking studies but is not the optimum for large biological or physical targets, dual-ring double scattering system was designed to produce irradiation fields of two sizes (i.e. 6 and $16 \mathrm{~cm}$ diameter) starting from a fix pencil beam at $148 \mathrm{MeV}$ and is also possible to obtain a modulation in depth adding a custom made range modulator, optimized to generate a $2.5 \mathrm{~cm}$ spread-out Bragg peak (SOBP). This device, detailed described in [10], is assembled when required in the zero degree beamline isocenter and is used since 2018 for biological irradiation on cells culture. In 2019 this set-up started to be used also for irradiation on electronic devices for SEU studies.
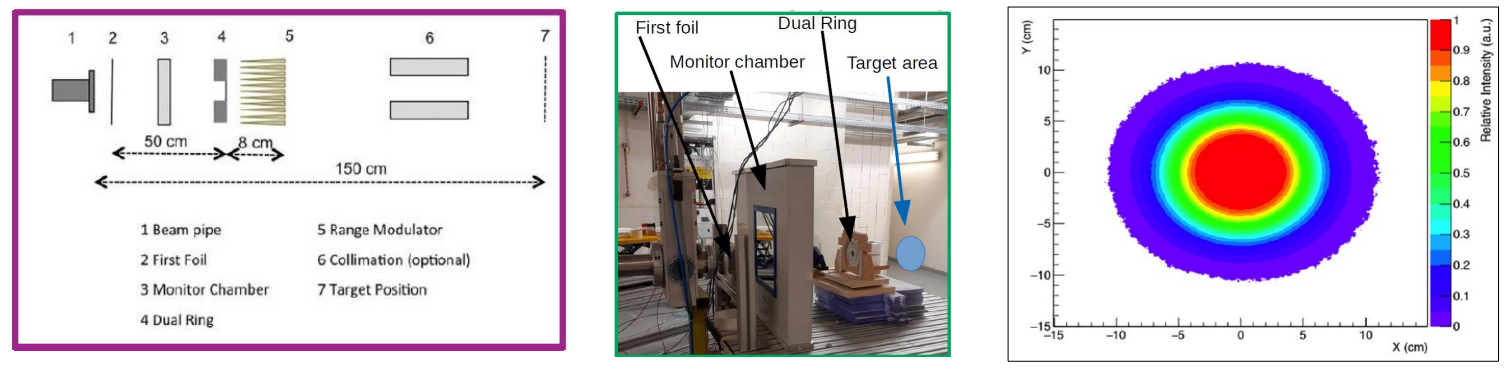

Fig. 2.2: dual ring schematic view (left), implementation (center) and measured output beam intensity profile (right).

Even if with the dual ring set-up the beam intensity is highly decreased by a factor 10 and 20, the beam obtained is extremely uniform on a circumference of large area, allowing uniform irradiations on large targets or single a irradiations on multiple small targets. 


\section{X-Ray irradiations}

In the TIFPA-INFN laboratories of the Povo site, irradiations can be performed also with an $\mathrm{x}$-ray source because are equipped with a Comet $3 \mathrm{KW}$ x-ray tube housed in a Xstrahl cabinet.

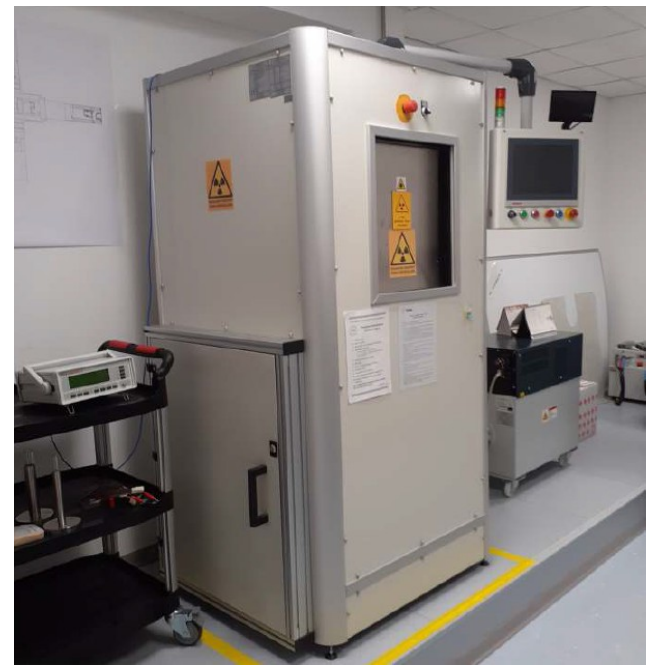

Fig. 3.1: The TIFPA-INFN x-ray irradiation cabinet in the Povo site laboratories.

The tube is realized with beryllium window and a tungsten anode where the anode voltage can be regulated from $195 \mathrm{kV}$ down to $30 \mathrm{kV}$, this allow to switch from tungsten K-alpha, K-beta to L-alfa, L-beta emission peaks in the x-ray spectrum.

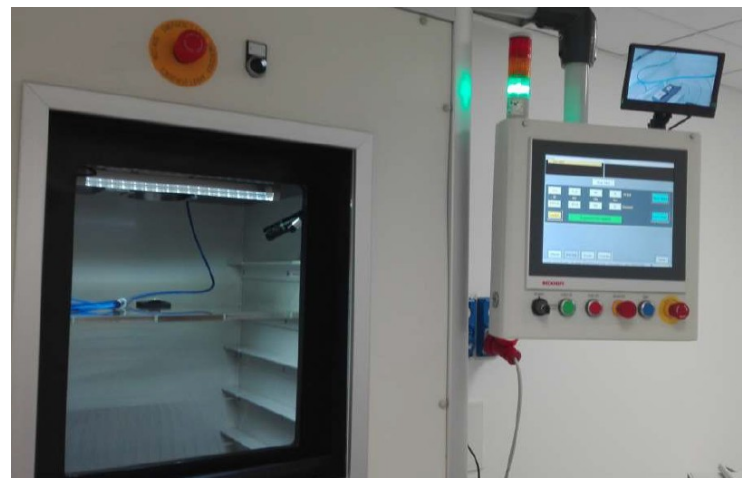

Fig. 3.2: The x-ray shielded irradiation volume. The x-ray tube is located on the top of the irradiation volume.

Anode current can be changed, according to the selected voltage, from $15 \mathrm{~mA}$ up to $30 \mathrm{~mA}$ Filters realized with different material (aluminum, copper) in different thickness can be changed by the users. Dose measurement system is composed by a calibrated PTW[11] Farmer Chamber read by a external electrometer. The target plane irradiation rate (and irradiated spot size) can be changed moving the support plane distance from the tube in the cabinet. The cabinet is also equipped with a laser spot-center pointer and a internal camera. The system was primarily build 
for biological irradiation on cells cultures but, due to its flexibility, since late 2019 is also used for irradiations on SiPM (designed in FBK[12]) for TID damage studies.
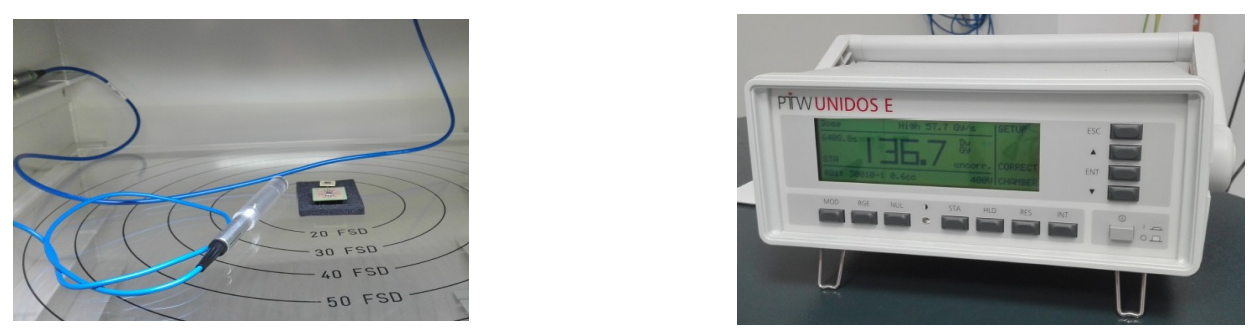

Fig. 3.3: The x-ray dose measurement system composed by a calibrated Farmer Chamber probe (left) and a PTW electrometer(right).

\section{Conclusions}

Proton and x-ray irradiations are essential studies required or the full characterization of a variety of solid state sensors, primarily silicon sensor but not only them. In the TIFPA-INFN laboratories there are the tools and the capabilities to perform these characterization, as demonstrated in this preliminary tests performed in 2019, and an increase of this this type of activity is expected in the next months.

\section{References}

[1] TIFPA-INFN web site: https://www.tifpa.infn.it

[2] Italian National Institute for Nuclear Physics: https://home.infn.it/en

[3] Trento Proton Therapy Center - TPTC (Trento, Italy):

https://protonterapia.provincia.tn.it/eng\#

[4] Azienda Provinciale per i Servizi Sanitari - APSS (Trento, Italy): https://www . apss . tn. it

[5] User beam time applications: http://www.tifpa.infn.it/sc-init/med-tech/pbeam-research

[6] Ion Beam Applications - IBA: https://iba-worldwide.com/proton-therapy

[7] Physics Department University of Trento: https://www.physics.unitn.it/en

[8] Yves Jongen, REVIEW ON CYCLOTRONS FOR CANCER THERAPY, Proceedings of CYCLOTRONS 2010, Lanzhou, China.

[9] F. Tommasino et al., Proton beam characterization in the experimental room of the Trento Proton Therapy facility, NIM A Volume 869, 11 October 2017, Pages 15-20.

[10] F. Tommasino et al., A new facility for proton radiobiology at the Trento proton therapy centre: Design and implementation, Physica Medica 58 (2019) 99-106.

[11] PTW The Dosimetry Company: https://www.ptwdosimetry.com/en

[12] Research Center Bruno Kessler Foundation - FBK (Trento): https: //www . fbk. eu/en 